\title{
étude expérimentale des fondations soumises au renversement
}

\author{
par \\ M. Ayeb, G. Biguenet, M. Dunand \\ Institut de Mécanique de Grenoble
}

\section{Introduction}

L'ingénieur constructeur est souvent amené à étudier des fondations sollicitées par des efforts inclinés. On rencontre ce problème pour les piles et culées de ponts, pour certains pieux ou groupes de pieux, et pour les ouvrages de soutènement. L'étude présentée ici étant plus particulièrement appliquée aux fondations des supports de lignes électriques, c'est-à-dire à des fondations parfaitement rigides à faible élancement. Elle a été réalisée à l'Institut de Mécanique de Grenoble, en collaboration avec la Direction des Études et Recherches d'E.D.F., division * Lignes aériennes:.

De nombreux essais en terrain naturel (sable propre, argile saturée, limon argileux), en semi-grandeur et en vraie grandeur, ont été réalisés dans le cadre de cette étude. Les mesures effectuées ont permis de déterminer expérimentalement le moment de renversement sollicitant la fondation, en fonction du déplacement angulaire de celle-ci, la répartition des contraintes à l'interface, et la cinématique du mouvement.

\section{Historique. Méthodes de calcul existantes}

Les méthodes de calcul des fondations soumises au renversement sont fort nombreuses. II n'est pas possible d'en donner une analyse exhaustive ici (on a pu analyser 35 méthodes différentes). [4 G. Berthomieu, 1976.] Ces méthodes peuvent être classées en 3 catégories :

- les méthodes dites élastiques, dans lesquelles les contraintes à l'interface sont liées aux déplacements de la fondation (Andree-Norsa, Sulzberger) [2,12] - les méthodes dites plastiques, dans lesquels on admet l'écoulement plastique dans tout le massif (Méthode française, Fröhlisch, Alexandru) [7, 1] - les méthodes expérimentales, ou élaborées à partir de l'observation de faits expérimentaux (Lazard, Dembicki, Méthode Belge, Méthode Menard) $9,5,10]$.
Les résultats des essais présentés ici ont été comparés à 5 méthodes classiques (Andree-Norsa, méthode française, Lazard, à la méthode Polonaise DembickiBolt-Odrobinski et à la méthode Menard).

\subsection{Méthode élastique: Andree-Norsa (fig. 1)} [2 Andree-Norsa 1966]

Les contraintes à l'interface sol-fondation sont normales, proportionnelles au déplacement, et au module de réaction du sol (K), lui-même proportionnel à la profondeur. En écrivant les équations d'équilibre, et grâce à diverses hypothèses simplificatrices, on aboutit à :

$$
M=P \cdot L / 2-2 \cdot P^{2} /(3 \cdot B \cdot p)+80 \cdot B^{2} D^{3} p^{2} /(6561 P)
$$

avec $\mathrm{M}$ : moment de renversement maximum de la fondation

$P$ : poids de la fondation et de la superstructure $B, L, D$ : largeur, longueur et hauteur de la fondation $p$ : pression maximum admissible

sous la base de la fondation, prise comme paramètre caractéristique du sol (calculé à partir de $\mathrm{C}$ et $\varphi$, ou déterminé par un essai en place).

Cette méthode, très simple donne des résultats acceptables dans les milieux pulvérulents. Par contre, elle est exagérément pessimiste dans les sols purement cohérents.

2.2 Méthode plastique : française, ou méthode du Réseau d'État (fig. 1) [7]

Les contraintes à l'interface sont proportionnelles à la profondeur suivant l'hypothèse classique de l'équilibre limite sur un écran vertical (pousséebutée). En écrivant les équations d'équilibre on obtient :

$$
M=P \cdot L / 2-2 \cdot P^{2} / 3 \cdot B \cdot P+B \cdot D^{3} \cdot A / 6
$$

les notations étant les mêmes que pour Andree-Norsa, avec : 


$$
A=\gamma \cdot\left[\operatorname{tg}^{2}(\pi / 4+\varphi / 2)-\operatorname{tg}^{2}(\pi / 4-\varphi / 2)\right]
$$

$\gamma$, poids volumique.

On adopte généralement, pour le terme $A / 6$, des valeurs corrigées d'après l'expérience qui vont de 0,28 (sol pulvérulent lâche) à $3,3 \cdot 10^{4} \mathrm{~N} / \mathrm{m}^{3}$ (terrain à forte cohésion).

\subsection{Méthode de Lazard [9]}

Elle constitue un aménagement de la méthode Belge (méthode Ramelot) [11] proposé par Lazard à l'issue de divers essais de renversement de supports de Lignes S.N.C.F. Une étude statistique sur les résultats d'essais en vraie grandeur a permis à Lazard de proposer l'expression suivante :

$$
M=\frac{10 D_{1}^{2} B}{H+D-\frac{D 1}{3}}
$$

avec $M$ : moment limite en mètre tonne

$H$ : hauteur de tirage en mètre

B : largeur des faces en butée, en mètres

D : fiche totale

D1: fiche "active"

$$
\text { en mètres }
$$

\subsection{Méthode Menard [10]}

Les contraintes à l'interface sont normales et peuvent croître jusqu'à la pression limite $\left(\mathrm{p}_{\ell}\right)$ pressiométrique. Menard distingue 2 cas, selon que l'effort de renversement est appliqué en tête de la fondation (moment négligé devant l'effort tranchant), ou à une certaine hauteur (enfort tranchant négligé devant le moment).

Dans le premier cas: $T=\alpha_{1} \cdot D \cdot h \cdot p_{\ell}$, moment négligeable.

Dans le second cas: $M=\alpha_{2} \cdot D \cdot h^{2} \cdot p_{\ell}$, effort tranchant négligeable avec

$T$ et $M$ : Effort horizontal en tête de la fondation et moment de renversement.

$\mathrm{D}$ et $\mathrm{h}$ : Diamètre et hauteur de la fondation.

$\mathrm{p}_{\ell}$ : Pression limite pressiométrique du sol.

$\alpha_{1}$ et $\alpha_{2}$ : Coefficients variables suivant la nature du sol et l'élancement de la fondation.

$$
0,2<\alpha_{1}<0,41 ; \quad 0,13<\alpha_{2}<0,25 \text {. }
$$

Les déplacements en tête du pieu dans la phase pseudo-élastique sont donnés par :

$$
\begin{aligned}
& K \cdot \operatorname{tg} \theta=\left(\alpha_{1} / 0,16\right) \cdot\left(p_{\ell} / h\right): 1^{\text {er }} \text { cas } \\
& K \cdot \operatorname{tg} \theta=\left(\alpha_{2} / 0,08\right) \cdot\left(p_{\ell} / h\right): 2^{e} \text { cas. }
\end{aligned}
$$

$\theta$ étant l'angle d'inclinaison du pieu et $\mathrm{K}$ le module de réaction du sol défini statistiquement à partir de $p_{\ell}$ dans le tableau suivant :

\section{Tableau 1}

\begin{tabular}{c|c|c|c}
\hline $\begin{array}{c}\text { Diamètre } \\
\text { du pieu }\end{array}$ & $0,3 \mathrm{~m}$ & $0,6 \mathrm{~m}$ & $1,2 \mathrm{~m}$ \\
\hline Argile & $\mathrm{p}_{\ell}=2 \mathrm{~K}$ & $\mathrm{p}_{\ell}=4 \mathrm{~K}$ & $\mathrm{p}_{\ell}=6 \mathrm{~K}$ \\
\hline Sable & $\mathrm{p}_{\ell}=\mathrm{K}$ & $\mathrm{p}_{\ell}=2 \mathrm{~K}$ & $\mathrm{p}_{\ell}=2,6 \mathrm{~K}$ \\
\hline
\end{tabular}

\subsection{Méthode polonaise (Dembicki-Bolt) [5]}

Élaborée par une équipe de chercheurs de l'Institut polytechnique de Gdansk à partir des résultats de nombreux essais sur modèles réduits, cette méthode

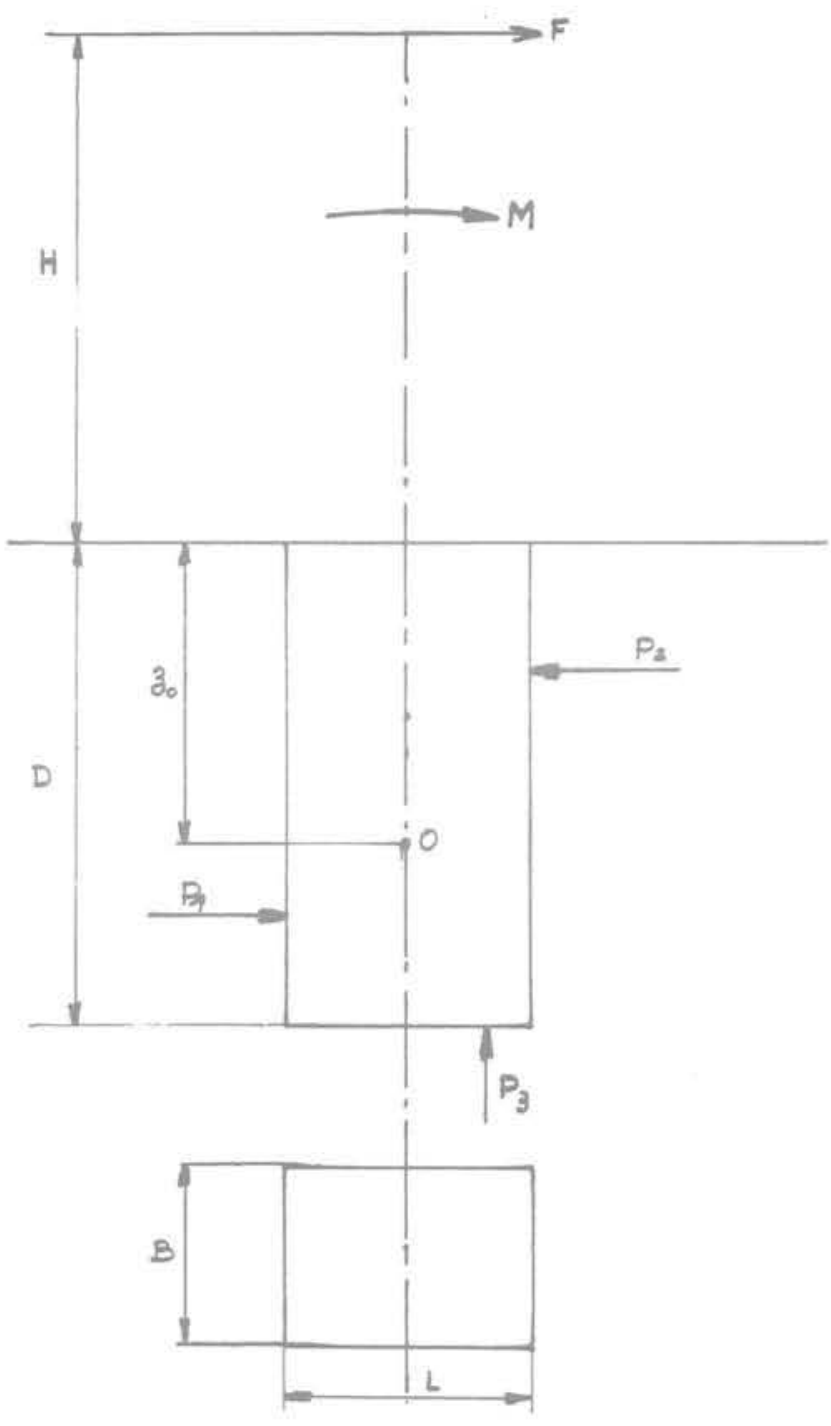

Fig. 1 Schéma de la fondation

s'applique aux fondations circulaires ou rectangulaires. On écrit les équations de l'équilibre statique en supposant aux interfaces une répartition de contraintes en plasticité parfaite (poussée-butée). Les auteurs ont présenté des abaques permettant d'obtenir le moment limite lorsque l'effort tranchant est faible, ou l'effort limite lorsque le moment est faible.

Cette méthode semble en assez bon accord avec l'expérience comme nous l'indiquons plus loin.

\subsection{Bilan des méthodes existantes. Répartition des contraintes à l'interface}

A l'issue d'une étude bibliographique complète (dont le texte ci-dessus est un très bref résumé), il apparaît que les méthodes existantes permettent d'obtenir le moment de renversement limite, mais pas les déplacements de la fondation (sauf, dans une certaine mesure, la méthode Menard). En outre, les hypothèses faites sur les répartitions de contraintes aux interfaces sol-fondation sont souvent arbitraires. En conséquence, les essais décrits ci-après avaient pour but de préciser les points suivants :

- répartition réelle des contraintes aux interfaces, - contrôle des déplacements de la fondation. 


\section{Processus et matériel d'essais}

Les essais de renversement ont été réalisés en terrain naturel, dans la région de Grenoble. Deux types de fondation ont été utilisés :

- Nondation rectangulaire de $0,4 \times 0,4 \times 0,8 \mathrm{~m}$ supportant un pylône de $5 \mathrm{~m}$ de hauteur (système dénommé : "petit pylône", figure 12).

- Fondation circulaire (diamètre $1 \mathrm{~m}$, fiche $3 \mathrm{~m}$ ) supportant un pylône de $12,5 \mathrm{~m}$ de hauteur (système dénommé "grand pylône ", figure 13).

\subsection{Les différents terrains d'essais}

Les sols naturels meubles dans lesquels se posent généralement les problèmes de fondation ont été symbolisés par 3 matériaux caractéristiques :

- un sable fin lavé

- une argile plastique saturée

- un limon sablo-argileux non saturé.

27 essais de renversement ont été réalisés dans ces 3 types de sols avec l'ensemble "petit pylónen, en 1980. En outre, 2 essais "grand pylônew ont été exécutés en 1981 dans un sol bicouche $(0$ à $2 \mathrm{~m}$ : limon argileux non saturé, 2 à $3 \mathrm{~m}$ : sable graveleux sous la nappe phréatique).

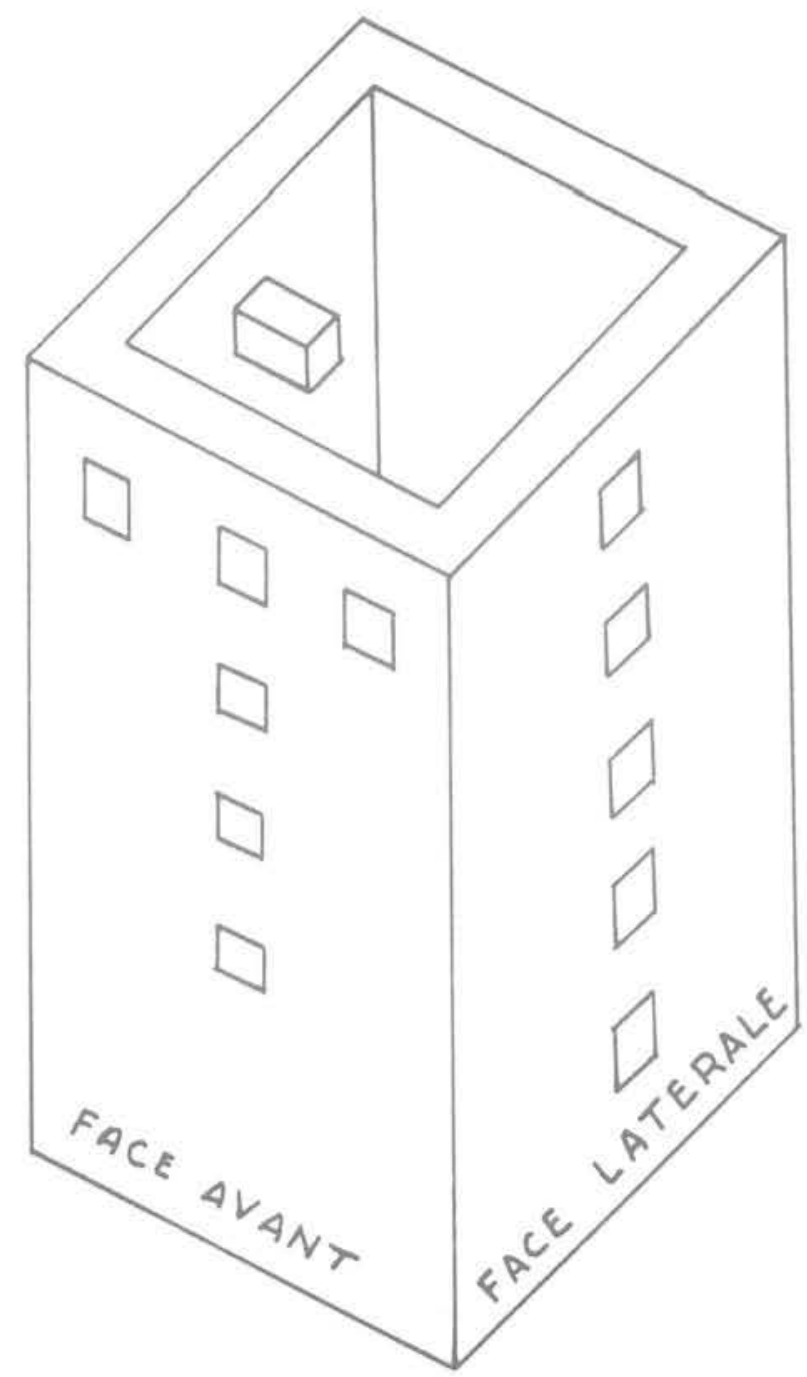

Fig. 2 Fondation (petit pylône) et capteurs
3.1.1 Essais dans le sable (petit pylône, 10 essais)

Afin de disposer d'un matériau parfaitement pulvérulent, il a été nécessaire de réaliser une fosse $(\mathrm{L}=5 \mathrm{~m}, \ell=3 \mathrm{~m}, \mathrm{~h}=2 \mathrm{~m}$ ) creusée à la pelle mécanique dans le sol naturel limoneux, et emplie de sable calibré lavé $\left(30 \mathrm{~m}^{3}\right)$.

\subsubsection{Essais dans l'argile (petit pylône : 5 essais)}

Situé à Varces, banlieue de Grenoble, dans une dépression, lacustre et marécageuse, ce terrain est constitué par une argile grise plastique saturée (nappe phréatique en surface), très homogène sur plus de $20 \mathrm{~m}$ d'épaisseur.

\subsubsection{Essais dans le limon (petit pylône : 12 essais)}

II s'agit des alluvions récentes de l'Isère. Situées à Saint-Martin d'Hères, à proximité du Laboratoire de Mécanique des Sols de I'Université de Grenoble. C'est un matériau fin, un peu argileux, contenant un sable très fin, et situé au-dessus de la nappe de l'Isère.

3.1.4 Essais en sol bi-couche (grand pylône: 2 essais)

Les essais "grand pylône" ont eu lieu également à proximité du laboratoire: la première couche est constituée par le limon décrit ci-dessus ( 0 à $2 \mathrm{~m}$ ), la seconde par un sable graveleux immergé (2 à $3 \mathrm{~m}$ ).

\subsubsection{Caractéristiques des sols étudiés}

Le tableau ci-après résume les résultats des analyses et mesures effectuées sur ces divers matériaux.

\subsection{Fondations utilisées}

\subsection{1 «Petit pylône"}

Les massifs de fondation sont en béton coulé en place, dans une fouille de $0,4 \times 0,4 \mathrm{~m}$ et $0,80 \mathrm{~m}$ de profondeur, pour les essais dans le limon (hors nappe). Ils sont préfabriqués et mis en place dans une fouille aux mêmes dimensions dans l'argile. Pour le sable $(c=0)$, le massif est mis en place par une grue dans une fouille talutée, et le sable est ensuite compacté uniformément autour du massif.

La fondation en béton est creuse, avec 5 parois de $5 \mathrm{~cm}$ d'épaisseur, comportant des réservations de $50 \mathrm{~mm}$ destinées à recevoir les capteurs de contrainte (voir fig. 2).

\subsection{2 "Grand pylône "}

La fondation est constituée par une virole en acier de $1 \mathrm{~m}$ de diamètre intérieur, $3 \mathrm{~m}$ de hauteur, $15 \mathrm{~mm}$ d'épaisseur, comportant également les réservations prévues pour les capteurs de contrainte. Les viroles sont foncées au marteau vibrant (3 tonnes), puis vidées à la benne preneuse.

\subsection{Système d'application des efforts}

- Les pylónes: lls sont en profilés acier (cornières et fer $\mathrm{U}$ ) du type treilli. Le petit pylône mesure $5 \mathrm{~m}$ de hauteur et $0,40 \mathrm{~m}$ à la base. Le grand pylône 
mesure $12,5 \mathrm{~m}$ de hauteur et $1 \mathrm{~m}$ à la base. Ils sont calculés pour recevoir en tête, sans déformation appréciable 1 tonne (petit pylône) et 10 tonnes (grand pylône). Après mise en place des fondations, les pylônes sont fixés sur celles-ci par boulons.

- Système de tirage: Un câble acier est fixé en tête du pylône par l'intermédiaire d'un capteur de force, et accroché, à l'autre extrémité, à un mouffle dont le brin actif s'enroule sur un treuil à moteur tournant à vitesse constante mais réglable. La vitesse de tirage est réglée de manière à obtenir une inclinaison du pylône de l'ordre de $15^{\circ}$ en 2 heures, afin de réaliser une mise en pression du sol quasi-statique.

Les fondations rectangulaires ont été renversées dans un plan perpendiculaire aux faces (et non suivant la diagonale).

\subsection{Mesure des contraintes aux interfaces}

Une série de 30 capteurs de contrainte tridimensionnels a été réalisée à l'Institut de Mécanique de Grenoble, suivant un modèle conçu et expérimenté sur plusieurs prototypes successifs. lis sont constitués par 3 étages de mesure destinés à mesurer chacun une des 3 composantes de la contrainte à l'interface. Chaque étage comporte 2 lames élastiques en acier disposées de telle sorte qu'elles fléchissent sous l'effet d'une composante de la contrainte, sans être affectées par les 2 autres composantes (lames d'acier à haute limite élastique de $1 \mathrm{~mm}$ d'épaisseur $20 \mathrm{~mm}$ de largeur et $40 \mathrm{~mm}$ de longueur). Quatre jauges de contraintes sont collées sur les lames élastiques ( 2 jauges par lame : une en compression et une en traction) et forment un point de Wheatstone complet pour chaque voie de mesure.

La face d'appui du capteur, en contact avec le sol, mesure $31,6 \times 31,6 \mathrm{~mm}\left(10 \mathrm{~cm}^{2}\right)$. Chaque étage de mesure peut recevoir un effort de $50 \mathrm{~kg}$ (pre sion à l'interface de 5 bars). L'ensemble est monté dans un boîtier en acier permettant sa fixation sur la fondation, à travers celle-ci de manière que la face d'appuí affleure l'interface sol-fondation. Chaque pont de mesure est alimenté par une tension continue de $6 \mathrm{~V}$, et fournit un signal de l'ordre de $3 \mathrm{mv}$ par volt pour 1 bar. Naturellement chaque voie de mesure de chaque capteur a été étalonnée sous charge statique, les valeurs ci-dessus sont des moyennes. Lors du dépouillement des mesures, l'étalonnage exact de chaque voie est pris en compte. (Voir fig. 14.)

\subsection{Mesure des déplacements de la fondation}

Afin de déterminer la cinématique du déplacement de l'ensemble fondation-pylône, on définit les trajectoires réelles de 3 points solidaires de la fondation à l'aide de 6 capteurs potentiométriques (voir fig. 3 ).

\subsection{Mesure et enregistrement des paramètres}

La chaine de mesure complète comprend: 60 capteurs, une alimentation stabilisée à $6 \mathrm{~V}$, une centrale de mesure HP 3495 A couplée à un calculateur HP $9825 \mathrm{~A}$. Les données (tension d'alimentation, 6 capteurs de déplacement, 52 capteurs de contraintes, effort de traction du cable) sont "scrutées" à raison d'un cycle par minute, traitées instantanément par le calculateur, enregistrées sur cassette, et imprimées. Ceci permet de suivre, en clair, l'évolution de tous les paramètres, cycle par cycle,

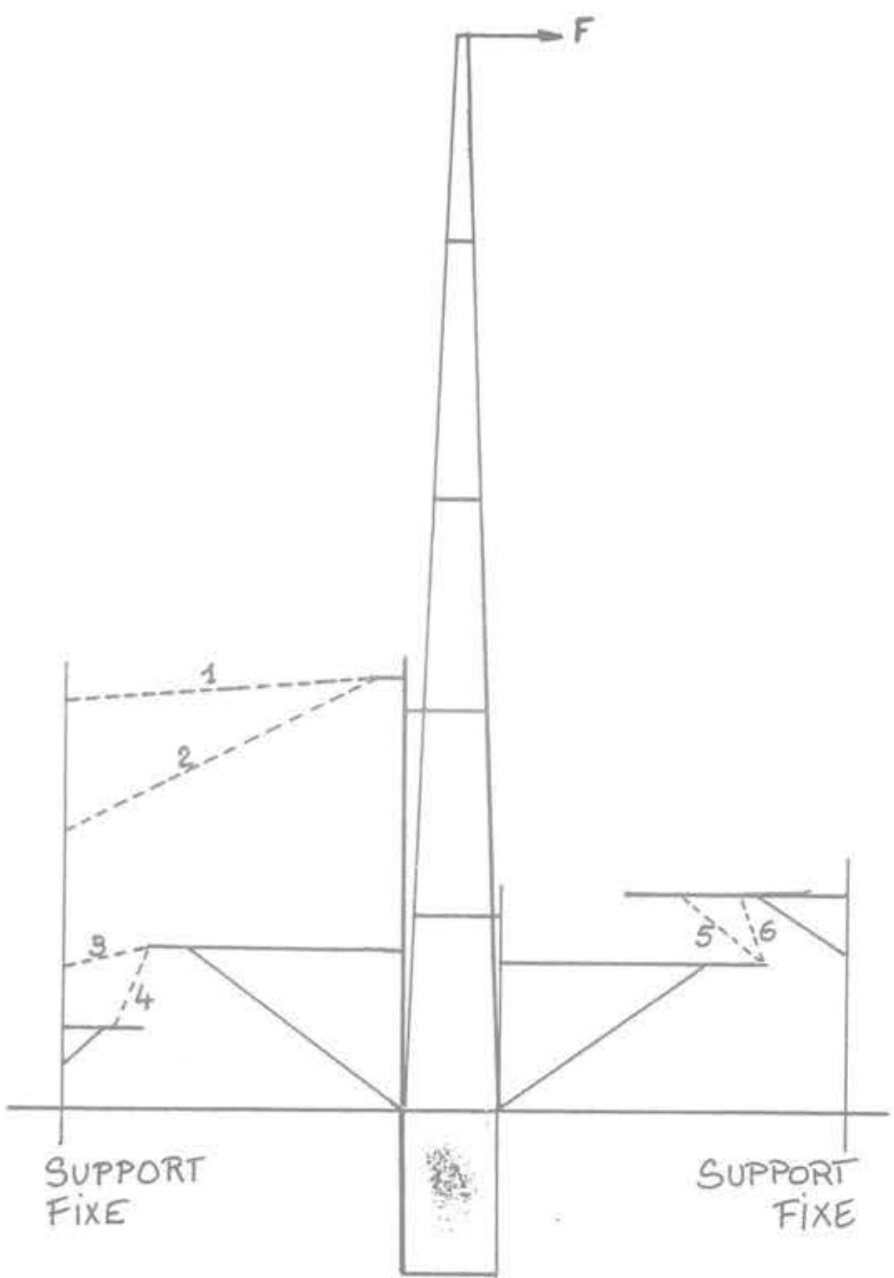

Fig. 3 Position des capteurs de déplacement

durant l'essai, et d'effectuer après coup diverses opérations sur les enregistrements (notamment, tracer l'évolution des contraintes en fonction des déplacements sur table traçante HP 9872).

\section{Résultats des mesures}

\subsection{Observations effectuées durant les essais de renversement}

Dans tous les essais on a pu observer tout d'abord un décollement de la fondation sur la face arrière au-dessus du centre de rotation, et au-dessous sur la face avant. Ce phénomène se produit même dans le sable propre, du fait d'une légère cohésion capillaire. On observe également des fissures à la surface du sol tout d'abord à l'avant de la fondation, puis à l'arrière. Ces fissures, très apparentes pour le petit pylône, le sont beaucoup moins pour le grand pylône. En outre, cette fissuration du sol en surface n'apparaît que pour une forte inclinaison, alors que le moment de renversement approche de sa valeur maximum. 


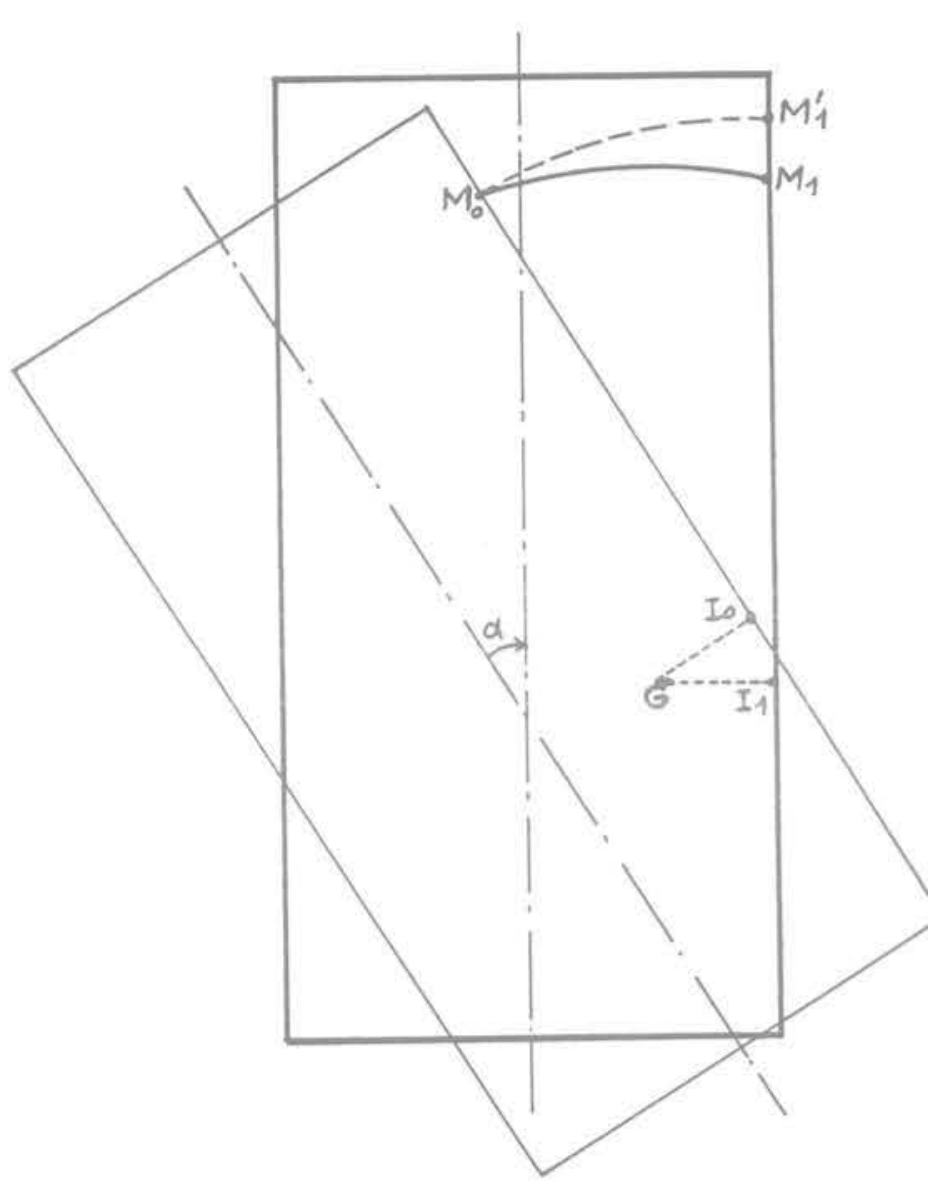

Fig. 4 Cinématique du renversement

\subsection{Cinématique du déplacement}

Le mouvement de l'ensemble fondation-pylône est un déplacement plan, défini par les trajectoires de 3 repères liés rigidement à la fondation. On peut définir ainsi à tout instant :

- le centre instantané de rotation (C.I.R.)

- le centre de rotation globale (C.R.G. : invariant dans la transformation qui fait passer le solide indéformable fondation-pylône de sa position initiale à une position quelconque).

La figure 4 illustre le mouvement de l'ensemble fondation-pylône.

La figure 5 indique les trajectoires du C. R. G. au cours de quelques essais.

\subsection{Valeur des contraintes mesurées à} l'interface

\subsubsection{Contrainte normale}

Elle est nulle au niveau du centre de rotation, et croit vers le haut en face avant et vers le bas en face arrière, jusqu'à atteindre une valeur très voisine de la pression limite obtenue au pressiomètre (fig. 6).

\subsubsection{Contraintes tangentielles}

Elles restent très faibles ( 5 à $10 \%$ des contraintes normales au même point de l'interface).
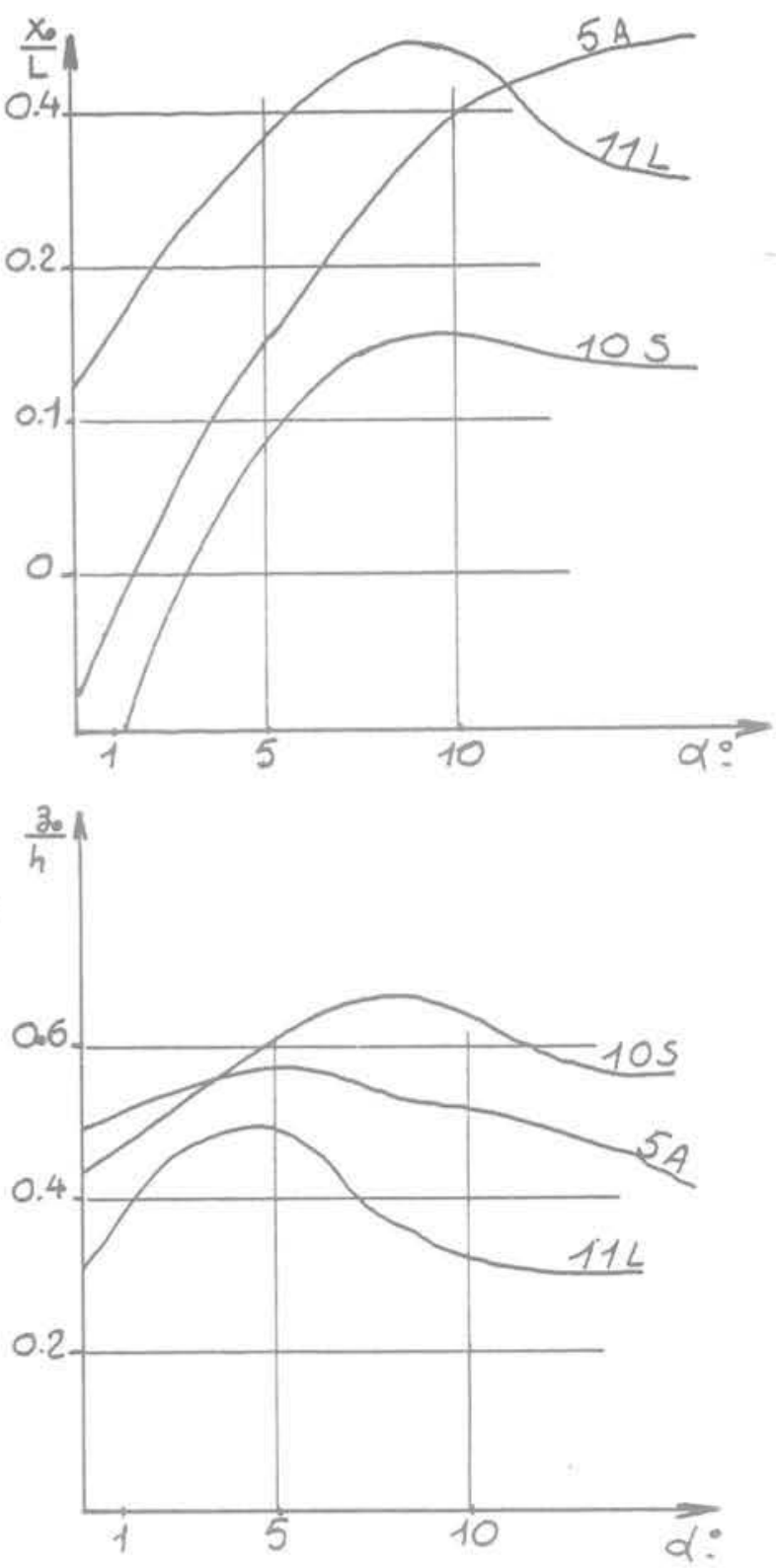

ESSAIS"PETIT PYLONE $\left\{\begin{array}{l}N^{\circ} 10 \text { dans le sable } \\ N^{*} 5 \text { dansl'argile } \\ N 11 \text { dans le limon }\end{array}\right.$

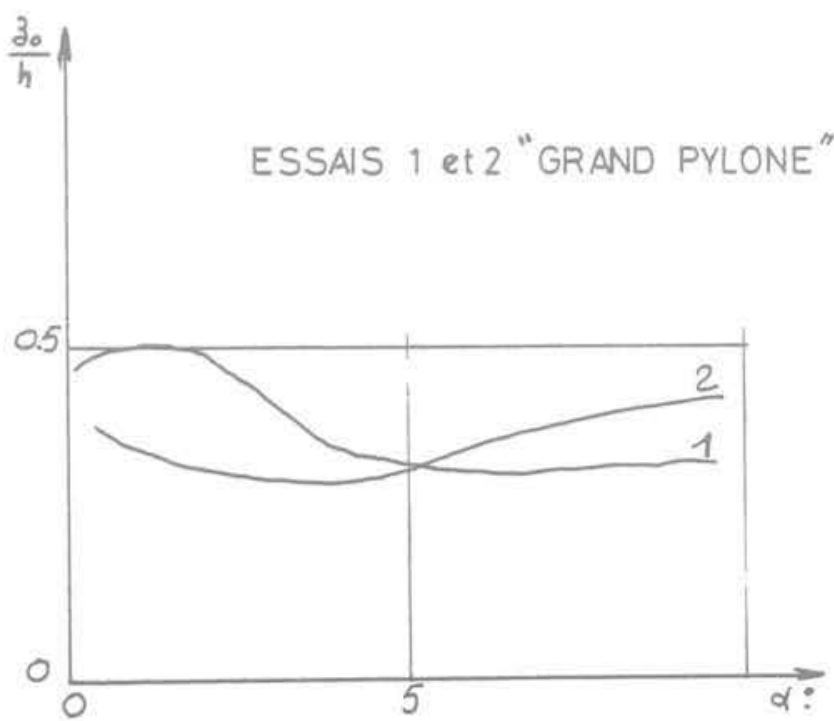

Fig. 5 Coordonnées du CRG en fonction de l'inclinaison 


\begin{tabular}{|c|c|c|c|c|c|c|c|c|c|}
\hline \multirow[t]{2}{*}{$\begin{array}{l}\text { Nature du } \\
\text { sol }\end{array}$} & \multirow{2}{*}{$\begin{array}{c}\text { Teneur en } \\
\text { eau naturelle } \\
\text { w } \%\end{array}$} & \multirow{2}{*}{$\begin{array}{c}\text { Masse } \\
\text { volumique } \\
\text { naturelie } \\
\mathrm{g} / \mathrm{cm}^{3}\end{array}$} & \multicolumn{2}{|c|}{$\begin{array}{l}\text { Cisaillement } \\
\text { direct }\end{array}$} & \multirow{2}{*}{$\begin{array}{c}\text { Vane test } \\
\stackrel{\mathrm{C}}{\mathrm{Pa} \times 10^{5}}\end{array}$} & \multicolumn{2}{|c|}{ Pressiomètre } & \multicolumn{2}{|c|}{ Valeurs moyennes } \\
\hline & & & $\varphi^{\circ}$ & $\underset{\mathrm{Pa} \times 10^{5}}{\mathrm{C}}$ & & $\begin{array}{l}\text { Module } \\
E M=2 G \\
P a \times 10^{5}\end{array}$ & $\begin{array}{c}\text { Pression } \\
\text { limite } \\
\text { Pa } \times 10^{5}\end{array}$ & $\begin{array}{c}2 \mathrm{G} \\
\mathrm{Pa} \times 10^{5}\end{array}$ & $\begin{array}{c}\mathrm{P}_{\ell} \\
\mathrm{Pa} \times 10^{5}\end{array}$ \\
\hline $\begin{array}{l}\text { Sable } \\
\text { lache }\end{array}$ & 2 & 1.57 & 36 & 0 & & 10 & $\begin{array}{l}1,2 \\
\text { à } \\
1,3\end{array}$ & 10 & 1,25 \\
\hline $\begin{array}{l}\text { Argile } \\
\text { saturée }\end{array}$ & 35 & 1,88 & 0 & 0.25 & 0,18 à 0,28 & 16 & $\begin{array}{c}1,8 \\
\grave{a} \\
2,25\end{array}$ & 16 & 2 \\
\hline $\begin{array}{l}\text { Limon } \\
\text { argileux }\end{array}$ & 33 & 1,85 & 20 & 0,3 & & $\begin{array}{l}26 \\
\text { à } \\
36\end{array}$ & $\begin{array}{c}3 \\
\text { a } \\
3,6\end{array}$ & 30 & 3,3 \\
\hline
\end{tabular}

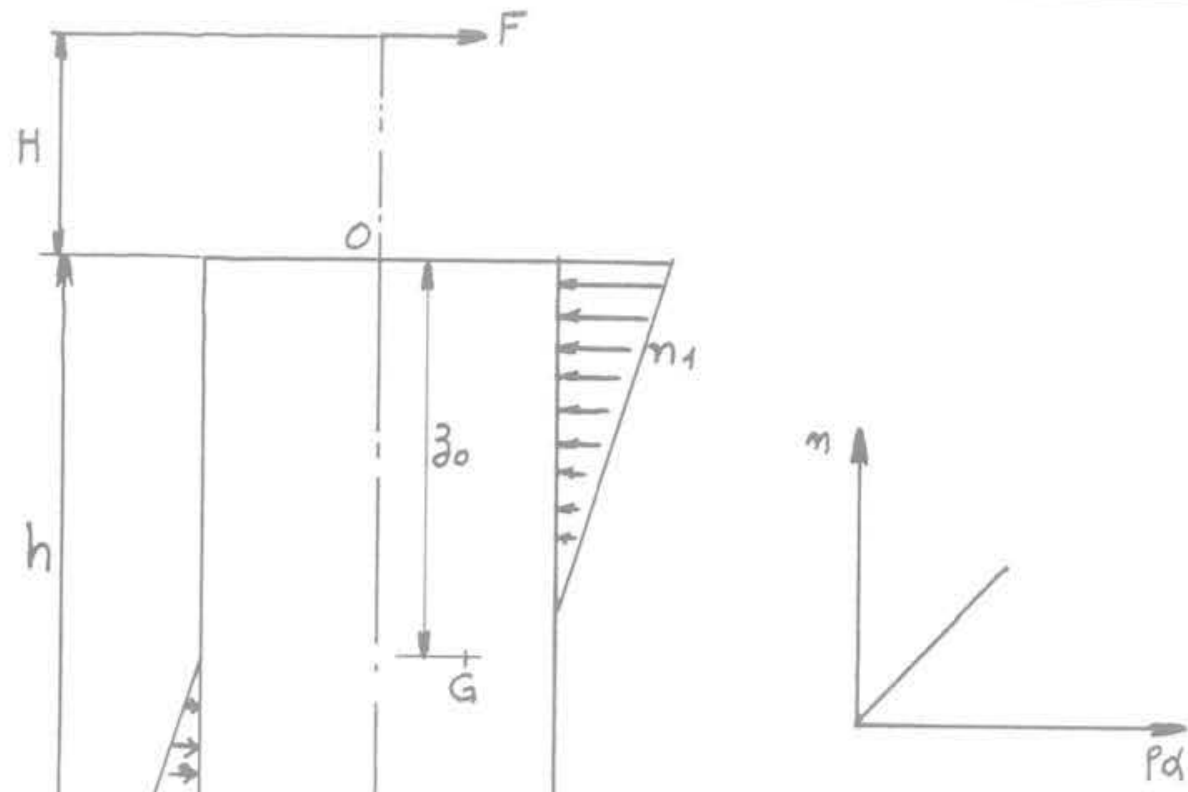

On observe ainsi une certaine dispersion des résultats, bien que tous les essais aient été conduits de la même manière dans des terrains apparemment homogènes.

Fig. 6 Distribution des contraintes - phase élastique

On a reporté sur les mêmes graphiques (fig. 8, 9, 10 et 11) les valeurs du moment limite calculé d'après diverses méthodes (Lazard, Bolt, André Norsa, Ménard, Méthode Française dite du Réseau d'état), ainsi que la courbe $F(\alpha)$ déterminée par la méthode I. M. G. exposée ci-après.

\subsection{Moment de renversement en fonction de l'angle d'inclinaison}

Pour tous les essais on dispose de la valeur de la force de tirage et de l'angle d'inclinaison enregistrés sur bande magnétique à partir des indications fournies par les capteurs (force et déplacements).

Les graphiques moment de renversement-angle d'inclinaison sont obtenus sur table traçante. Compte tenu du nombre important d'essais effectués, nous avons reporté sur les figures $8,9,10$ et 11 les courbes enveloppes des graphiques expérimentaux. Ces dernières s'inscrivent toutes à l'intérieur du fuseau représenté en grisé.

\section{Méthode de calcul proposée}

En fonction des observations faites durant les essais et sommairement rapportées ci-dessus, il est possible de proposer les hypothèses suivantes:

- la cinématique du renversement peut être assimilée à un déplacement plan défini par l'axe commun du support et de sa fonction et par la direction de l'effort de tirage;

- la composante normale de la contrainte à l'interface augmente linéairement à partir de la cote du centre de rotation jusqu'à atteindre la valeur he la pression limite prise égale à la pression limite pressiométrique; 

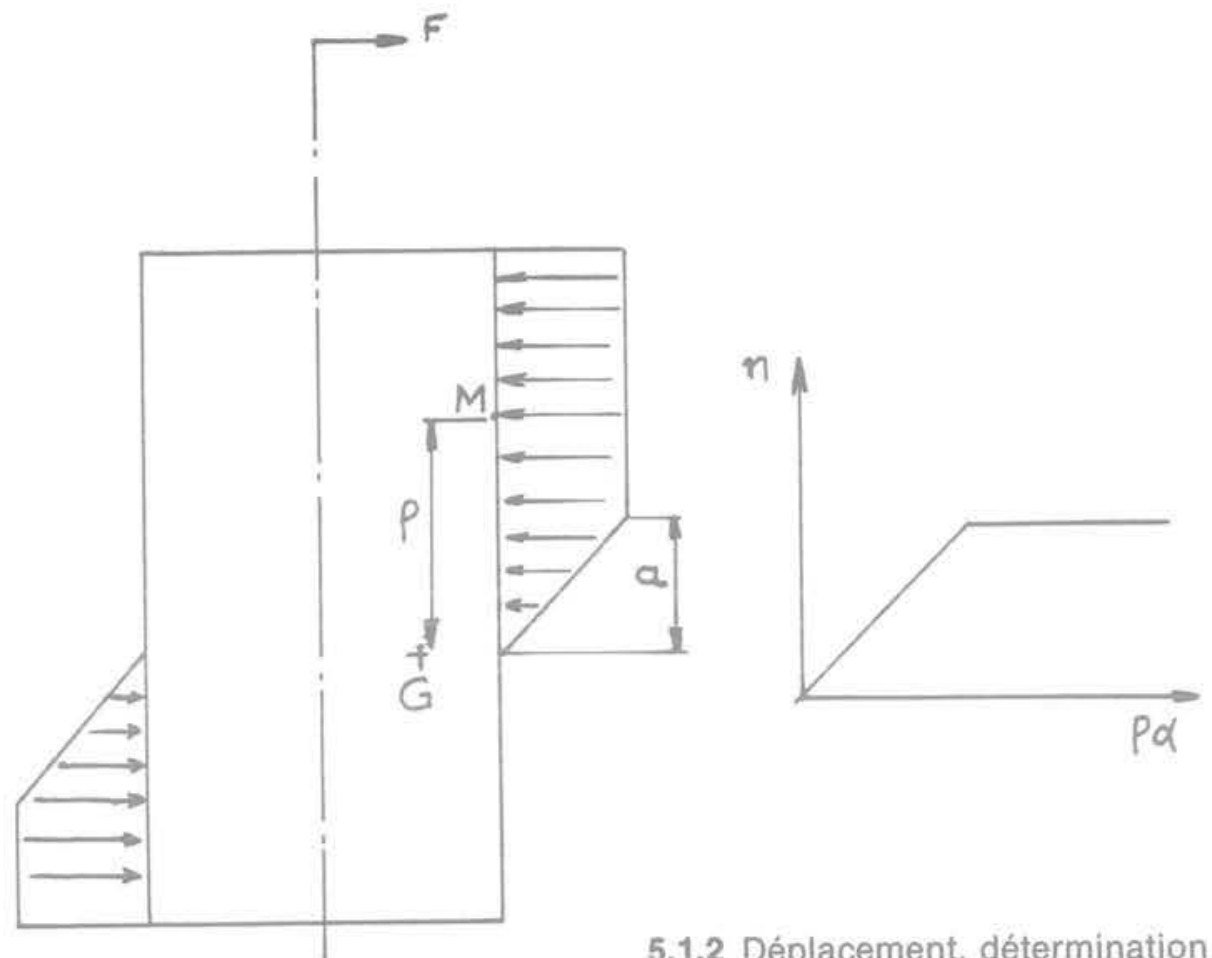

Fig. 7 Phase élastique-plastique

- la fondation est rigide, le sol est homogène et admet une loi de comportement élastique, parfaitement plastique.

\subsection{Phase élastique}

Au début du mouvement de renversement, lorsque l'effort $F$ est faible les contraintes normales $n_{1}$ et $n_{2}$ sont inférieures à la pression limite $\mathrm{p}_{\ell}$. Les déplacements à l'interface sont donc liés linéairement aux contraintes. En utilisant les résultats des calculs de J. P. Giroud [8], on peut exprimer les déplacements en fonction des caractéristiques élastiques du sol (G et $\gamma$ par exemple).

\subsection{1 Équations d'équilibre de la fondation} (fig. 6)

On admet que le poids est équilibré par les pressions normales sous la base et par les contraintes tangentielles le long du fût.

$$
\begin{aligned}
& F=2 b\left[\frac{z_{0} \cdot n_{1}}{2}-\frac{\left(h-z_{0}\right) n^{2}}{2}\right] \\
& 2 b \frac{z_{0} n_{1}}{2}\left(H+\frac{z_{0}}{3}\right)=2 b \cdot \frac{\left(h-z_{0}\right) n_{2}}{2}\left(H+z_{0}+\frac{2}{3} h-\frac{2}{3} z_{0}\right) .
\end{aligned}
$$

Les contraintes normales maximum sont alors :

$n_{1}$ (surface du sol en face avant) $=K \cdot z_{0} \cdot \alpha$.

$\mathrm{n}_{2}$ (base de la fondation en face arrière) $=\mathrm{K}\left(\mathrm{h}-\mathrm{z}_{0}\right) \alpha$ avec $\mathrm{K}$ : module de réaction du sol.

$$
\alpha \text { : inclinaison de la fondation. }
$$

On trouve :

$$
\mathrm{z}_{\mathrm{O}}=\frac{\mathrm{h}(3 \mathrm{H}+2 \mathrm{~h})}{3(2 \mathrm{H}+\mathrm{h})}
$$

constant durant toute la phase élastique

$$
F=\frac{b \cdot h^{3}}{3(2 H+h)} \cdot K \cdot \alpha \text {. }
$$

5.1.2 Déplacement, détermination du module de réaction

Le déplacement d'une fondation rigide soumise à un effort excentré est (d'après J. P. Giroud) :

$$
W_{M}=\frac{1-v^{2}}{E} \cdot \frac{F}{h \cdot 2 b}\left[2 b \cdot \bar{P}+z \cdot e_{z} \bar{Q}\right]
$$

$\bar{P}$ et $\bar{Q}$ sont des coefficients ne dépendant que du rapport $\frac{h}{2 b}$.

$e_{2}$ est l'excentricité de l'effort, soit ici

$$
e_{z}=\frac{H+\frac{h}{2}}{h}
$$

Le déplacement du bord supérieur de la fondation sera alors :

$$
\mathrm{z}_{\mathrm{o}} \alpha=\frac{1-v}{2 \mathrm{G}} \cdot \frac{\mathrm{F}}{\mathrm{h} \cdot 2 \mathrm{~b}}\left[2 \mathrm{~b} \cdot \overline{\mathrm{P}}+\left(\frac{\mathrm{H}}{2}+\frac{\mathrm{h}}{4}\right) \overline{\mathrm{Q}}\right]
$$

soit

$$
\alpha \cdot \frac{h(3 H+2 h)}{3(2 H+h)}=\frac{1-v}{2 G} \cdot \frac{h^{2} \cdot K \cdot \alpha}{6(2 H+h)}\left[2 b \cdot \bar{P}+\left(\frac{H}{2}+\frac{h}{4}\right) \bar{Q}\right]
$$

d'où

$$
K=\frac{2 G}{1-v} \cdot \frac{3 H+2 h}{2 b \cdot h} \cdot \frac{2}{\bar{P}+\frac{2 H+h}{8 b} \bar{Q}}
$$

$\mathrm{K}$ ne dépend que de la géométrie du système et du facteur $2 G /(1-v)$.

\subsection{Phase élastique-plastique}

Lorsqu'apparaît la plasticité (obligatoirement au niveau du sol) on a :

$$
\mathrm{n}_{1}=\mathrm{K} \cdot \mathrm{z}_{0} \cdot \alpha_{\mathrm{c}}=\mathrm{p}_{\ell}
$$

ce qui définit $\alpha_{c}$ (inclinaison critique) $=\frac{\mathrm{p}_{\ell}}{\mathrm{K} \cdot \mathrm{z}_{0}}$. En partant du centre de rotation la contrainte normale $n$ est proportionnelle à $\left(z_{0}-z\right)$ jusqu'à une distance "a " du centre de rotation; au-delà de $\mathrm{a}, \mathrm{n}$ reste constante et égale à $\mathrm{p}_{\ell}$. 


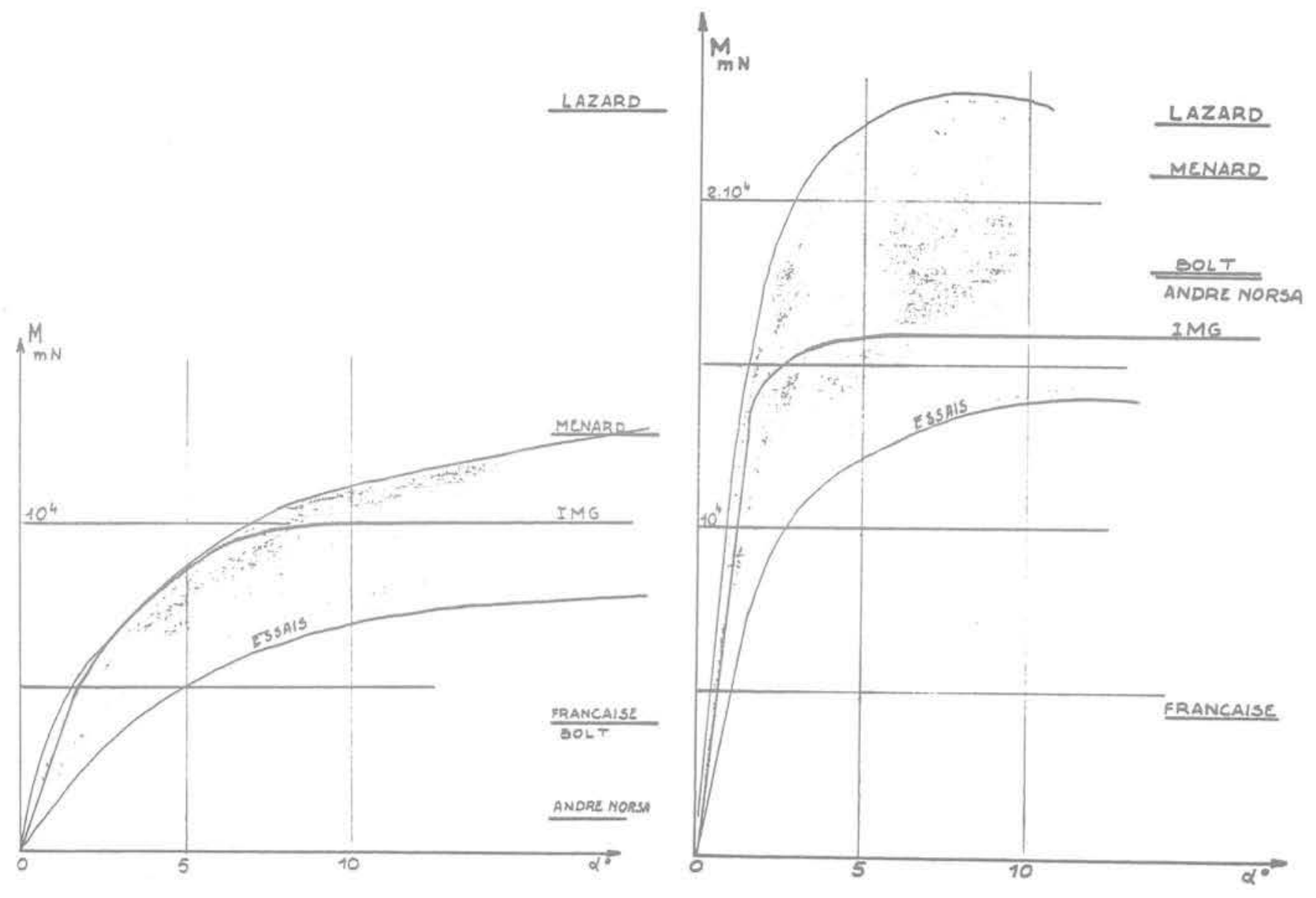

Fig. 8 Petit pylône-argile

Fig. 9 Petit pylône - limon
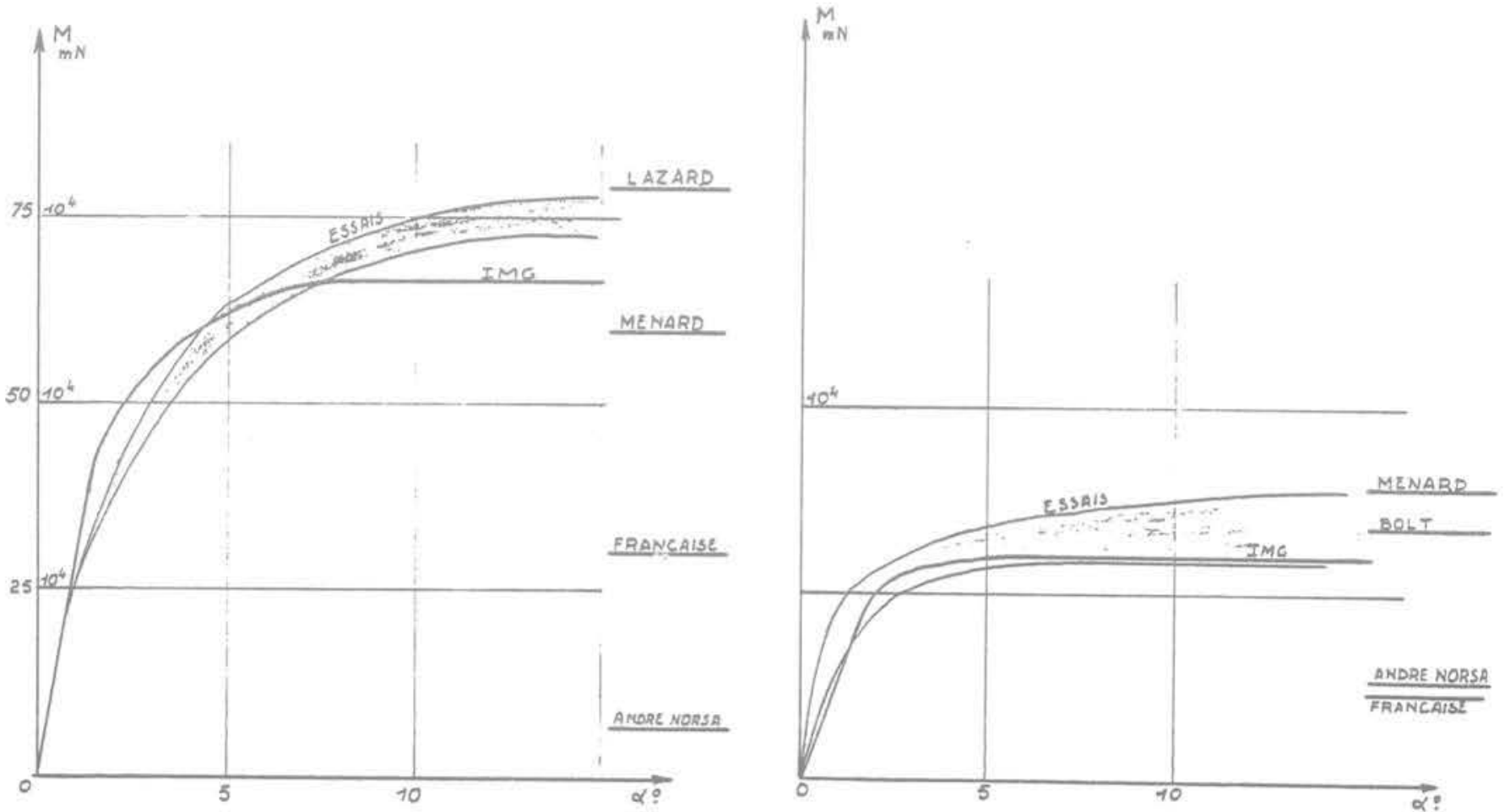

Fig. 10 Grand pylône - limon

Fig. 11 Petit pylône - sable 
CABLE DE

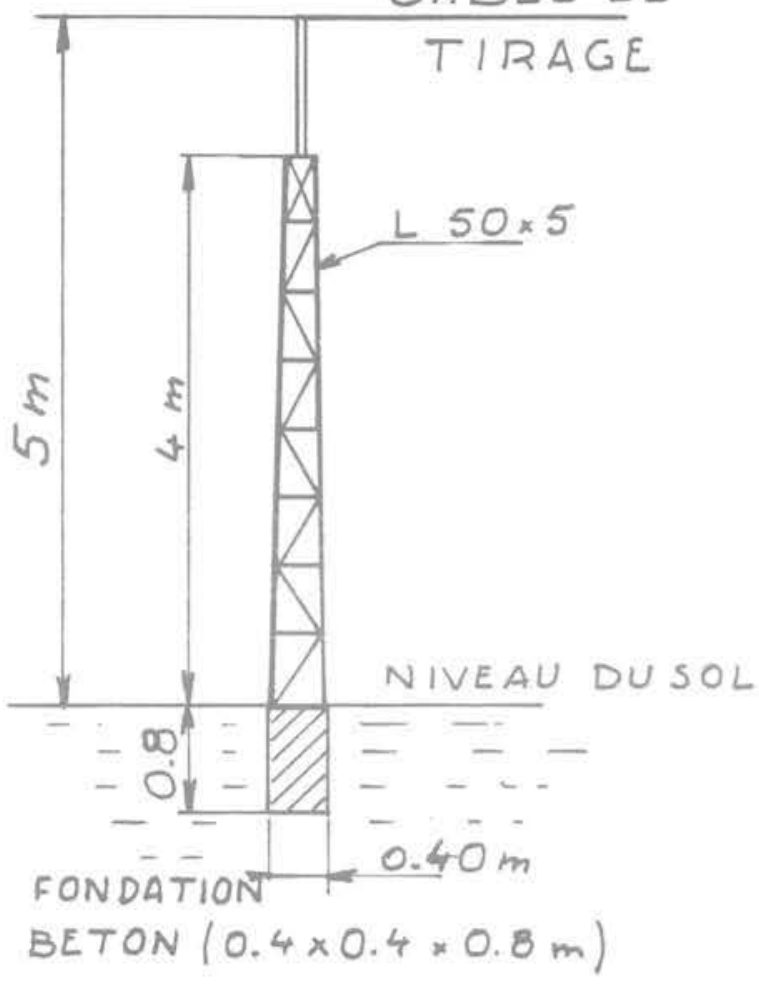

Fig. 12 Petit pylône (échelle 0.02)

Calcul de a : rayon critique.

On a

$$
a \cdot \alpha=\frac{p_{\ell}}{K} \quad \text { soit } \quad a=\frac{p_{\ell}}{K \alpha} .
$$

\subsection{1 Équations d'équilibre (fig. 7)}

- Forces verticales : les contraintes sous la base de la fondation et les composantes tangentielles verticales équilibrent le poids du massif et de sa structure.

- Forces horizontales :

$$
\frac{F}{2 b \cdot p_{e}}=\left(z_{0}-a\right)+\frac{a}{2}-\left(h-z_{0}-a\right)+\frac{a}{2}
$$

(1) soit $\frac{F}{2 b \cdot p_{e}}=2 z_{0}-h$.

\section{- Moments :}

$\frac{F\left(H+z_{0}\right)}{2 b \cdot p_{e}}=\left(z_{0}-a\right)\left(\frac{z_{0}-a}{2}+a\right)+\frac{a}{2} \cdot \frac{2 a}{3}+\left(h-z_{0}-a\right)$

$$
\left(\frac{h-z_{0}-a}{2}+a\right)+\frac{a}{2} \cdot \frac{2 a}{3}
$$

(2) soit $\frac{F\left(H+z_{0}\right)}{2 b \cdot p_{e}}=\frac{3 z_{0}^{2}+3\left(h-z_{0}\right)^{2}-2 a^{2}}{6\left(H+z_{0}\right)}$.

Les 2 relations (1) et (2) permettent de calculer $z_{0}$ et $F$. On trouve :

$$
\begin{aligned}
& z_{0}=\sqrt{H^{2}+H h+\frac{h^{2}}{2}-\frac{a^{2}}{3}}-H \\
& F=2 b \cdot p_{e}\left(2 z_{0}-h\right) .
\end{aligned}
$$

\subsection{Récapitulation}

Au début du mouvement (phase élastique) on a : $\mathrm{F}=\mathrm{A} \cdot \boldsymbol{\alpha}$

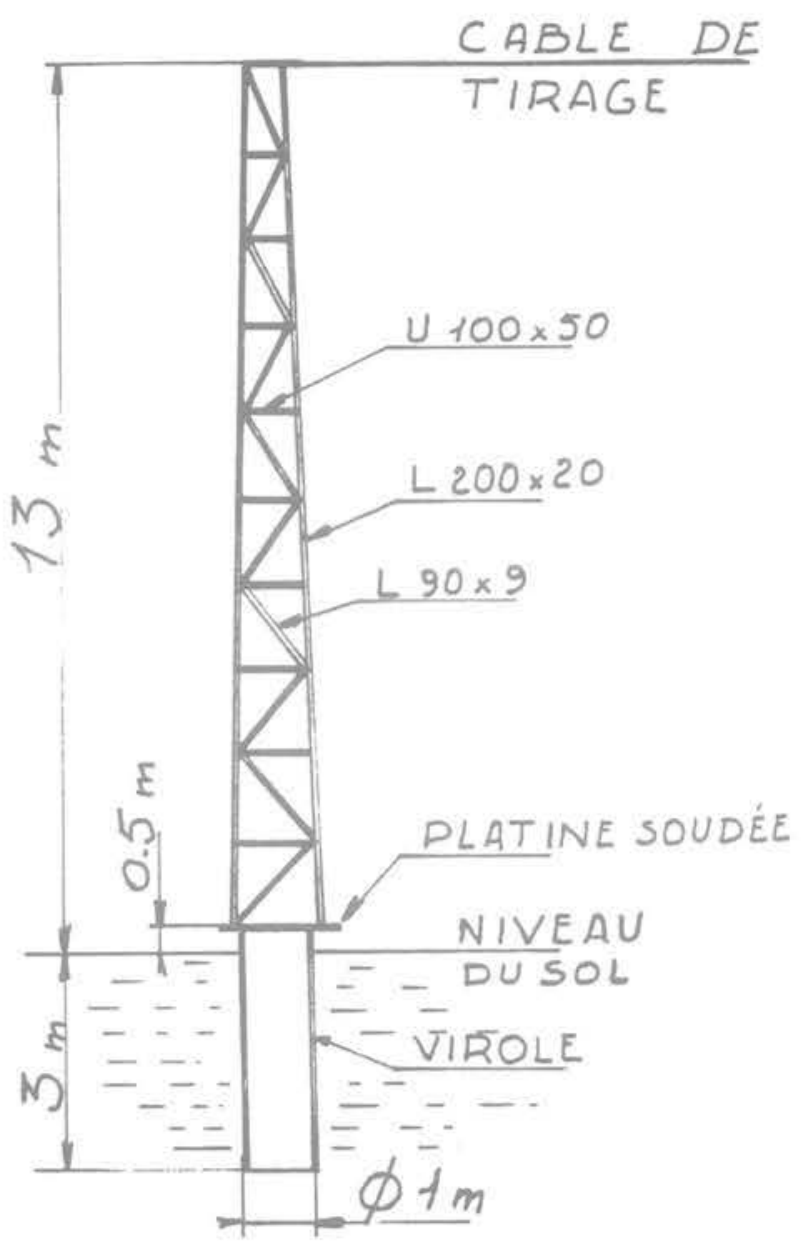

Fig. 13 Grand pylône (échelle 0.01)

$\alpha$ : angle de rotation de la fondation (en radians) $A=h^{2} \cdot \frac{A_{1} \cdot A_{2}}{A_{3} \cdot A_{4}}$

$A_{1}=\frac{2 G}{1-v} ; A_{2}=2 h+3 H ; A_{3}=3(h+2 H) ;$

$A_{4}=\bar{P}+\frac{2 H+h}{8 b} \cdot \bar{Q}$

$P$ et $Q$ : coefficients adimensionnels donnés par les tables.

Lorsque $\alpha$ dépasse la valeur critique de telle sorte que :

$$
\alpha_{c}=\frac{P_{\ell}}{A_{1}} \cdot \frac{b \cdot A_{3}}{\left(A_{2}\right)^{2}} \cdot A_{4}
$$

la fonction $F(\alpha)$ n'est plus linéaire, et on a :

$$
\begin{aligned}
F & =2 b \cdot p_{e}\left(2 z_{0}-h\right) \\
z_{0}+H & =\sqrt{H^{2}+H h+\frac{h^{2}}{2}-\frac{a^{2}}{3}} \\
a & =\frac{p_{\ell}}{A_{1}} \cdot \frac{h}{A_{2}} \cdot A_{4} \cdot \frac{b}{\alpha}
\end{aligned}
$$

5.4 Application aux essais

5.4.1 Caractéristiques pressiométriques moyennes des sols étudiés

(On admettra pour tous les sols : $\nu=1 / 3$.)

- Sable : $p_{\ell}=1,25 \cdot 10^{5} \mathrm{~Pa}$.

Module de cisaillement $2 \mathrm{G}=10^{6} \mathrm{~Pa}$ 


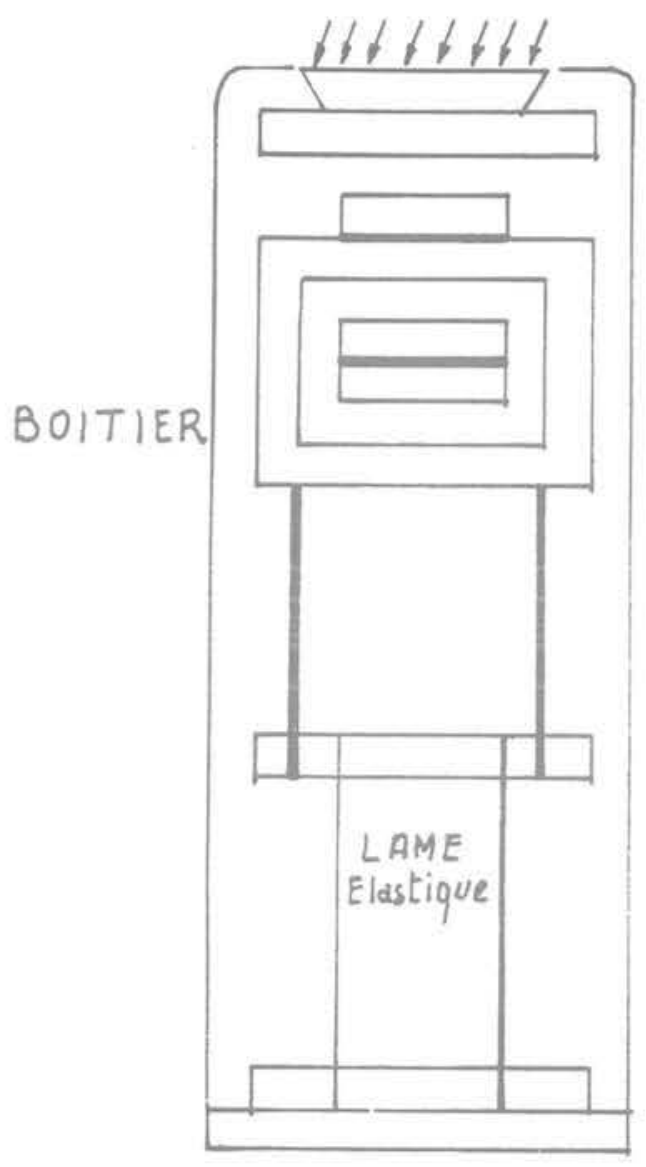

FACE

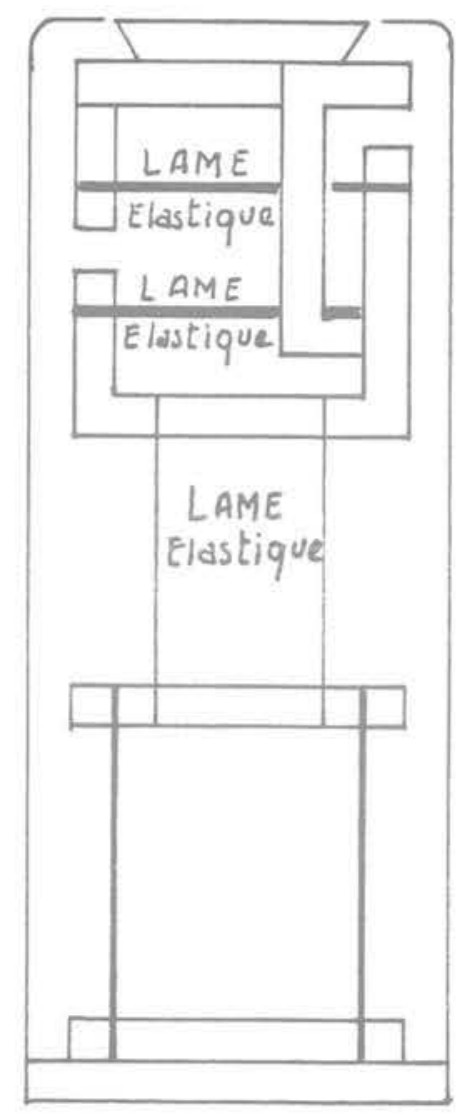

Etage 2:

FORCE TANGENCIELLE $\mathrm{H}$

Etage 3 :

FORCE TANGENTIELLE

Fig. 14 Capteur de contrainte tridimensionnel (échelle 1)

d'où $A_{1}=15 \cdot 10^{5} \mathrm{~Pa}$.

- Argile: $\mathrm{p}_{\ell}=2 \cdot 10^{5} \mathrm{~Pa}$

$$
2 \mathrm{G}=16 \cdot 10^{5} \mathrm{~Pa} \text {, soit } \mathrm{A}_{1}=24 \cdot 10^{5} \mathrm{~Pa} \text {. }
$$

- Limon : $p_{\ell}=3,3 \cdot 10^{5} \mathrm{~Pa}$

$$
2 \mathrm{G}=30 \cdot 10^{5} \mathrm{~Pa} \text {, soit } \mathrm{A}_{1}=45 \cdot 10^{5} \mathrm{~Pa} .
$$

\subsubsection{Données géométriques}

- Petit Pylône : $2 \mathrm{~b}=0,43 \mathrm{~m} ; \mathrm{h}=0,76 \mathrm{~m} ; \mathrm{H}=4,90 \mathrm{~m}$ valeur des tables pour $\frac{h}{2 b}=1,77\left(\right.$ soit $\left.\frac{2 b}{h}=0,57\right)$ :

$$
\bar{P}=1,15 \text { et } \bar{Q}=3,57
$$

d'où : $\quad A_{2}=15,22 \mathrm{~m} ; \quad A_{3}=31,68 \mathrm{~m}$;

$$
A_{4}=12,11 \mathrm{~m} \text {. }
$$

- Grand Pylône : $2 \mathrm{~b}=1 \mathrm{~m} ; \mathrm{h}=3 \mathrm{~m} ; \mathrm{H}=13 \mathrm{~m}$ valeur des tables pour $\frac{h}{2 b}=3\left(\right.$ soit $\left.\frac{2 b}{h}=1 / 3\right)$ :

$$
\begin{array}{cl}
\bar{P}=1,43 \quad \text { et } \quad \bar{Q}=2,95 \\
\text { d'où } & A_{2}=45 \mathrm{~m} ; \quad A_{3}=58 \mathrm{~m} ; \\
A_{4}=12,12 .
\end{array}
$$

\subsubsection{Résultats}

a) Petit Pylóne

- Essais dans le sable :

$$
\alpha_{c}=0,0297 r \text { soit } 1,7 \text { degré }
$$$$
A=0,344 \cdot 10^{5} \mathrm{~N} \text { et } F_{c}=A \alpha_{c}=1021 \mathrm{~N}
$$

d'où $\quad \mathrm{M}=5000 \mathrm{mN}$

$F_{\text {Lim }}$ (limite théorique pour $\left.\alpha \longrightarrow \infty\right)=1236 \mathrm{~N}$

et $\quad \mathrm{M}=6080 \mathrm{mN}$.

- Essais dans l'argile :

$$
\begin{gathered}
\alpha_{c}=0,029 \mathrm{r} \quad \text { soit } 1,7 \text { degré } \\
A=0,55 \cdot 10^{5} \mathrm{~N} \quad \text { et } F_{c}=A \cdot \alpha_{c}=1017 \mathrm{~N} \\
M_{c}=4983 \mathrm{mN} \\
F_{\mathrm{lim}}=2036 \mathrm{~N} ; \quad M=9976 \mathrm{mN} .
\end{gathered}
$$

- Essais dans le limon:

$$
\begin{gathered}
\alpha_{c}=0,261 \mathrm{r} \quad \text { soit } 1,5 \text { degré } \\
A=1,031 \cdot 10^{5} \mathrm{~N} \quad \text { et } \quad F_{c}=2691 \mathrm{~N} \\
M_{c}=13186 \mathrm{mN} \\
F_{\text {lim }}=3264 \mathrm{~N} ; \quad M=15994 \mathrm{mN} .
\end{gathered}
$$




\section{b) Grand Pylône}

Essais dans le limon

$$
\begin{gathered}
\alpha_{\mathrm{c}}=0,0255 \mathrm{r} \quad \text { soit } 1,46 \text { degré } \\
\mathrm{A}=25,926 \cdot 10^{5} \mathrm{~N} \quad \text { et } \quad F_{\mathrm{c}}=32926 \mathrm{~N} \\
\mathrm{M}_{\mathrm{c}}=428038 \mathrm{mN} \\
\mathrm{F}_{\mathrm{lim}}=51070 \mathrm{~N} ; \quad \mathrm{M}=663910 \mathrm{mN} .
\end{gathered}
$$

Les valeurs numériques ci-dessus permettent de tracer la courbe représentant la fonction $F(\alpha)$.

Les figures $8,9,10$ et 11 montrent l'écart entre les valeurs théoriques et les valeurs données par l'expérience.

\section{Conclusion}

L'Institut de Mécanique de Grenoble a procédé à de nombreux essais de renversement de fondations en terrain naturel, en collaboration avec E.D.F. Les mesures effectuées ont porté sur le moment de renversement en fonction de l'angle d'inclinaison sur la répartition des contraintes le long de l'interface sol-fondation, et sur la cinématique du mouvement. La méthode de calcul proposée ici, déduite des observations faites durant les expériences, permet de prévoir la variation du moment de renversement en fonction de l'angle d'inclinaison, à partir des résultats d'essais pressiométriques. Le calcul donne des valeurs en assez bonne concordance avec l'expérience.

\section{Références bibliographiques}

[1] Alexandru V. (1970): "Contribution au calcul des fondations encastrées pour les pylônes des lignes électriques aériennes». Institut de la Construction, Bucarest.
[2] Andrée-Norsa (1966), "Calcul des massifs». Groupe de travail "Supports de lignes", E.D.F.G.D.F.

[3] Ayeb M. (1983), "Étude des fondations soumises au renversement. Application du Pressiomètre". Thèse de Docteur-Ingénieur présentée le 13 janvier 1983, à l'Université de Grenoble.

[4] Berthomieu G. (1976), "Modèle tridimensionnel de fondations de pylônes sollicités horizontalement n. Thèse de Docteur de Spécialité présentée le 21 septembre 1981 à I'Université de Grenoble.

[5] Dembicki, Odrobinski, Bolt (1971), "Stability of single foundations columns $\%$. Varsovie-Podznan.

[6] Dunand M. (1981), "Étude expérimentale du comportement des fondations soumises au renversement ". Thèse de Docteur-Ingénieur présentée le 24 -mars 1981 à l'Université de Grenoble.

[7] Fayoux P. (1952), "Fondations des pylônes des lignes électriques à très haute tension \#. Bulletin de la Société Française des Électriciens.

[8] Giroud J. P. (1972), "Tables pour le calcul des fondations». Vol. 2 pages 201 à 210. Publié chez Dunod.

[9] Lazard A. (1955), "Moment limite de renversement de fondations cylindriques et parallélépipédiques isolés». A.I.T.B.T.P. tome $8 n^{\circ} 85$ (janv. 1955).

[10] Ménard L. (1962), a Comportement d'une fondation profonde soumise à des efforts de renversement $n$. Sols-soils, vol. $n^{\circ} 3,1962$.

[11] Ramelot-Vandeperre (1950), Comptes rendus de recherches I.R.S.I.A. $n^{\circ} 2$.

[12] Sulzberger G. (1945), "Les fondations de supports de lignes aériennes et leur calcul . Association Suisse des Électriciens, $\mathrm{n}^{\circ} 10$ (mai 1945). 
